\title{
Evaluation of Central and Peripheral Corneal Thicknesses in Patients with Systemic Lupus Erythematosus
}

This article was published in the following Dove Press journal: Clinical Ophthalmology

\section{Iman M Eissa $\mathbb{D}$ \\ Ghada A Nassar \\ Shaimaa A Arfeen \\ Ahmed A Dahab (D)}

Department of Ophthalmology, Cairo University, Giza, Egypt
Correspondence: Ahmed A Dahab Cairo University, 27 Madinat Al-

Mabouthin, Giza, Egypt

Tel +96655923।865

Email dr.adahab@yahoo.com
Purpose: To evaluate the corneal pachymetric and topographic parameters of systemic Lupus Erythematosus (SLE) patients using Dual Scheimpflug Imaging.

Methods: This observational cross-sectional controlled study included the right eye of 30 SLE patients and 30 age-matched controls. Corneal measurements were acquired by dual Scheimpflug imaging including anterior and posterior corneal curvatures, central, midperipheral corneal thickness (measured at the $5 \mathrm{~mm}$ zone) and peripheral pachymetry (measured at the $7 \mathrm{~mm}$ zone). SLE disease activity index (SLEDAI) was calculated and correlated with corneal pachymetry.

Results: SLE patients had significantly thicker corneal periphery than controls. Mean central corneal pachymetry was $530.4 \pm 27.3$ microns (SD) in SLE and $547.5 \pm 31.5$ microns (SD) in control group, $\mathrm{p}=0.032$. The corneal periphery - except superiorly - was significantly thicker in SLE patients than controls $(\mathrm{p}<0.001)$. Nasal peripheral corneal thickness positively correlated with disease activity index SLEDAI ( $\mathrm{p}=0.03$ ).

Conclusion: SLE patients present with thicker corneal periphery than controls characteristically sparing the superior quadrant. Possible corneal photosensitivity leading to peripheral immune complex deposition as well as flatter posterior corneal surface at the periphery are proposed explanations for these findings.

Keywords: cornea, corneal pachymetry, corneal topography, SLEDAI score, systemic lupus erythematosus

Systemic Lupus Erythematosus (SLE) is a chronic, autoimmune, multiple organ system connective tissue disease with a relapsing remitting course. The disease usually starts around late teens to early forties with females being affected 9 times more than males. The disease course and outcome vary between industrialized and developing countries. ${ }^{1,2}$

Around one third of SLE patients develop ocular manifestations. ${ }^{3}$ Keratoconjunctivitis sicca is the most common ocular manifestation of SLE, with its hallmark being decreased production of the aqueous layer of the tear film. Other more serious ocular manifestations include episcleritis, scleritis, retinal vasculitis (which is frequently associated with CNS lupus) and papillitis.,

The corneal affection in SLE may occur secondary to keratoconjunctivitis sicca as corneal epitheliopathy, filamentary keratitis, scarring and ulceration. Rarely, peripheral ulcerative keratitis (which can be a marker of active systemic vasculitis), interstitial keratitis as well as endotheliitis (dysfunctional appearing corneal endothelial cells) were reported to occur in SLE patients. ${ }^{5,6}$ 
Corneal biomechanical properties were reported to differ in SLE. In a study done by Yazici et al in 2011 found corneal hysteresis and corneal resistance factor to be lower in SLE patients than controls as measured by Reichert ocular response analyzer ORA (Reichert Ophthalmic Instruments, Buffalo, NY, USA). This could lead to underestimated IOP. Some authors relate the development of keratoconus to certain immune disorders as well. ${ }^{7,8}$

Having reviewed these studies, the authors found there was little information concerning the cornea as a refractive structure in lupus patients. Thus, this study examines the possibility of 'corneal parameter changes' in active lupus patients using one of the currently most reliable techniques for corneal evaluation; the Dual Scheimpflug Imaging.

Lupus patients are also well known to have photosensitivity. Exposure to sunlight not only triggers the characteristic skin rash, but also triggers systemic disease activity such as fatigue and arthralgia. ${ }^{9}$ Being an ultraviolet ray (UVR) sensitive structure in normal subjects, it is not yet known whether the cornea of lupus patients would react to sunlight exposure in a different or exaggerated pattern than normal subjects or not.

Lupus patients with refractive errors may also demand to do LASIK or other refractive procedures. The ophthalmologist should be acquainted with possible corneal pachymetric and topographic changes; to be able to make surgical decisions or recommendations.

\section{Patients and Methods}

The study was performed following the tenets of the Declaration of Helsinki. A verbal informed consent was obtained from all contributing subjects and was approved by the ethics committee of the Ophthalmology department, Cairo University.

This cross-sectional controlled study took place between May 2017 and June 2018 at the Ophthalmology department, Cairo University. Egypt. SLE patients were consecutively recruited from the Rheumatology and rehabilitation Department, Cairo University. Control subjects included patients coming to the ophthalmology outpatient clinic for routine eye checkup and some volunteering relatives of patients who had no ocular complaint. The rheumatology department receives around 8 new SLE patients per month, besides old follow up cases. Patients confirmed to have SLE according to the revised American College of Rheumatology (ACR) diagnostic criteria for $\mathrm{SLE}^{10}$ were included in the study.

We included the right eye in all patients for statistical analysis. Initially, the authors examined 46 cases of active SLE; of which sixteen cases were later excluded from the study during the examination process. Seven patients had severe dry eye which would have affected the Scheimpflug image quality, 2 had steroid-induced glaucoma, 3 were cognitively impaired due to CNS lupus and thus could not provide a valid consent and four were contact lens wearers. Finally, the study included 30 eyes of 30 patients with active SLE and 30 eyes of 30 age and sex-matched controls.

Exclusion criteria included severe dry eye (Schirmer test less than $5 \mathrm{~mm}$ ), history of ocular trauma or surgery, contact lens use, any corneal infiltration seen by slit-lamp examination, glaucoma, diabetes mellitus and any refractive error $> \pm 4 \mathrm{D}$ spherical equivalent (to avoid possible corneal thickness changes with refractive error)

\section{Examination}

The age, gender and the duration of disease for all patients were recorded. Systemic lupus disease activity index (SLEDAI) was assessed by consultants from the rheumatology department one day prior to ophthalmic evaluation. Based on the SLEDAI score, disease activity was categorized as follows: no activity (SLEDAI 0), mild activity (SLEDAI 1-5), moderate activity (SLEDAI 6-10), high activity (SLEDAI 11-19), and very high activity (SLEDAI $\geq 20$ ). ${ }^{11}$

\section{Ophthalmologic Examination}

All subjects underwent a detailed ophthalmic examination including a medical history review and best-corrected visual acuity (BCVA) using Snellen chart. The anterior segment was examined by slit-lamp biomicroscopy. Intraocular pressure (IOP) was measured using Goldmann applanation tonometry. The fundus was examined with a 90D lens, and any pathologic retinal findings were recorded.

\section{Dry Eye Evaluation and Scheimpflug Imaging} Evaluation of dry eye was performed prior to Scheimpflug imaging to prevent inaccurate pachymetric measurements. ${ }^{12}$ Corneal fluorescein staining was evaluated under cobalt blue light after fluorescein instillation. Subsequently, Schirmer's test was performed with topical anesthesia. Two minutes after one drop of proparacaine $0.5 \%$ was instilled, a Schirmer's test strip was placed at the lower lid margin's outer third. After $5 \mathrm{~min}$, the strip was removed, and the wet portion measured in millimeters. According to Schirmer test score, eyes with severe dry eye (less than $5 \mathrm{~mm}$ of wetting) were excluded from the study in order not to affect pachymetric measurements.

Corneal parameters were then examined. To avoid possible diurnal variation in corneal measurements, corneal imaging was performed within the same time interval 
(from 11 am to $1 \mathrm{pm}$ ), and at a temperature that ranged from 23 to $27{ }^{\circ} \mathrm{C}$ and relative humidity of $30 \%$ to $45 \%{ }^{13}$

Corneal parameters were evaluated with Sirius camera (Costruzione Strumenti Oftalmici; CSO, Florence, Italy, CSO, Sirius) which uses a combination of a Placido disk and a rotating Scheimpflug camera to measure corneal curvature and the corneal thickness across the whole cornea. Eyes of all contributing subjects were scanned by the same experienced and masked ophthalmologist. The measurement automatically started when correct alignment and focus of the eyes were achieved.

The corneal parameters of SLE group and control group were recorded. These included: central average pachymetry (central corneal thickness CCT), thinnest pachymetry (thinnest location), mid-peripheral corneal thickness (measured at the $5 \mathrm{~mm}$ zone) as well as peripheral corneal thickness (measured at the $7 \mathrm{~mm}$ zone) in microns. Mid-peripheral as well as peripheral corneal thickness were taken at the superior, inferior, nasal and temporal points, at 12, 6, 3 and 9 O'clock positions. Anterior and posterior corneal power at the flatter $(\mathrm{K} 1, \mathrm{rf})$ and the steeper $(\mathrm{K} 2, \mathrm{rs})$ corneal meridia were also recorded for each subject.

\section{Statistical Analysis}

Data were statistically described in terms of mean \pm standard deviation $( \pm \mathrm{SD})$, median and range, or frequencies (number of cases) and percentages when appropriate. Comparison of numerical variables between the study groups was done using Student $t$-test for independent samples. For comparing gender, Chi-square $\left(\chi^{2}\right)$ test was performed. Correlation between SLEDAI and various variables was done using Spearman rank correlation equation. $\mathrm{P}$ values less than 0.05 were considered statistically significant. All statistical calculations were done using computer program IBM SPSS (Statistical Package for the Social Science; IBM Corp, Armonk, NY, USA) release 22 for Microsoft Windows.

\section{Results}

\section{Descriptive and Comparative Statistics Duration, Age and Sex Distribution}

60 subjects were enrolled in this study, 30 in the SLE (study) group, and 30 in the control group. The mean duration of SLE in the study group was $6.8 \pm 5.4$ years.

Mean age in the SLE group was $30.2 \pm 9.1$ SD, while in the control group it was $34.2 \pm 9$ SD. The difference in age was not statistically significant $(\mathrm{p}=0.1)$.
In the SLE group, $83.33 \%$ were females while $16.66 \%$ were males. In the Control group, $76.66 \%$ were females and $23.33 \%$ were males. The difference in sex distribution among the two groups was not statistically significant.

\section{Ophthalmologic Examination}

Seven eyes among the examined patients had cataract $(23.3 \%)$. One patient with retinal vasculitis (3.3\%). As regards dry eye, 8 eyes $(26.6 \%)$ showed moderate dryness and 22 eyes had mild dryness $(73.3 \%)$ according to schirmer's test.

\section{Average and Thinnest Central Pachymetry}

The mean average central corneal thickness (CCT) in the SLE group was $530.4 \pm 27.3$ microns, while in the control group it was $547.5 \pm 31.5$ microns. The difference between both groups was statistically significant; with SLE patients having thinner average central pachymetry $(p=0.032)$. The average thinnest pachymetry was $525.45 \pm 30.25$ microns in the SLE group and $544.03 \pm 31.2$ microns in the control group. The difference was again statistically significant $(p=0.025)$. Logistic regression was done to adjust for gender as a possible confounder in comparison between cases and control regarding CCT, the $\mathrm{p}$ value was found to be statistically insignificant $(\mathrm{p}=0.09)$.

\section{Anterior and Posterior Corneal Curvatures (D)}

The values of the anterior corneal power in the flat (K1) and steep (K2) meridia were: SLE K1 (43.35 \pm 1.8 D), Control K1 (43.93 \pm 1.3 D), SLE K2 (44.39 \pm 1.9 D), Control K2 (44.92 \pm 1.4 D). All these parameters did not show a statistically significant difference between the two groups $(\mathrm{p}=0.17, \mathrm{p}=0.25, \mathrm{p}=0.59$, respectively).

For the posterior corneal curvature (in diopters), the flat $(\mathrm{K} 1 / \mathrm{rf})$ and steep $(\mathrm{K} 2 / \mathrm{rs})$ meridian within the $8 \mathrm{~mm}$ zone were measured. A statistically significant difference was found between the two groups with SLE patients having flatter posterior corneal surface than controls (Table 1). $\mathrm{P}$ value was 0.03 for the flat meridian (K1), and 0.02 for the steep (K2) meridian.

Table I Posterior Corneal Curvature Within the $8 \mathrm{Mm}$ Zone in the Two Groups

\begin{tabular}{|l|l|l|l|}
\hline & Rf(D) Mean \pm SD & Rs(D) Mean \pm SD & P value \\
\hline SLE & $-6.15 \pm 0.33$ & $-6.43 \pm 0.23$ & 0.03 \\
Control & $-6.34 \pm 0.36$ & $-6.60 \pm 0.28$ & \\
\hline
\end{tabular}

Abbreviations: Rf, radius flat (diopter); Rs, radius steep (diopter). 
Table 2 Mean Pachymetry Readings (in Microns) at Center, MidPeriphery and Periphery

\begin{tabular}{|l|l|l|l|l|l|}
\hline \multirow{2}{*}{} & \multicolumn{3}{l}{ Group } & \\
\cline { 2 - 6 } & \multicolumn{2}{|l|}{ SLE Group } & \multicolumn{2}{l|}{ Control Group } & P value \\
\cline { 2 - 6 } & Mean & $\begin{array}{l}\text { Standard } \\
\text { Deviation }\end{array}$ & Mean & $\begin{array}{l}\text { Standard } \\
\text { Deviation }\end{array}$ & \\
\hline Age & 30.28 & 9.19 & 34.28 & 9.08 & 0.101 \\
Thinnest & 525.45 & 30.25 & 544.03 & 31.21 & 0.025 \\
location & & & & & \\
SM (at 5 mm) & 611.07 & 35.27 & 596.97 & 34.56 & 0.130 \\
NM (at 5 mm) & 586.97 & 39.11 & 579.24 & 32.95 & 0.419 \\
IM (at 5 mm) & 577.72 & 37.63 & 573.21 & 29.76 & 0.614 \\
TM (at 5 mm) & 571.93 & 38.02 & 576.90 & 39.06 & 0.626 \\
SP (at 7 mm) & 666.69 & 49.06 & 658.31 & 38.93 & 0.474 \\
NP (at 7 mm) & 686.76 & 45.32 & 631.69 & 36.98 & $<0.001$ \\
IP (at 7 mm) & 679.14 & 41.34 & 619.93 & 32.95 & $<0.001$ \\
TP (at 7 mm) & 661.07 & 43.62 & 633.45 & 42.94 & 0.018 \\
CCT & 530.45 & 27.39 & 547.55 & 31.51 & 0.032 \\
\hline
\end{tabular}

Abbreviations: SM, superior mid-periphery; NM, nasal mid-periphery; IM, inferior mid-periphery; TM, temporal mid-periphery; SP, superior periphery; NP, nasal periphery; IP, inferior periphery; CCT, central corneal thickness.
The corneal topographic shape was as follows: 16 regular corneas, 9 symmetric bow tie, and 5 asymmetric bow tie patterns. No specific shape abnormalities were detected.

\section{Corneal Thickness at Mid Periphery}

In the SLE group: The mean corneal thickness at the superior mid-peripheral (SM) point was $611 \pm 35.2$ microns, nasal mid periphery (NM) was $586.9 \pm 39.1$ microns, inferior mid periphery (IM) was $577.7 \pm 37.6$ microns, and the temporal mid periphery (TM) was $571.9 \pm 38$ microns.

In the control group: The mean corneal mid-peripheral thickness at $\mathrm{SM}$ point was $596.9 \pm 34.5$ microns, $\mathrm{NM}$ was $579.2 \pm 32.9$ microns, IM was $573.2 \pm 29.7$ microns, and TM was $576.9 \pm 39$ microns (Table 2).

The difference between corresponding mid-peripheral points in both groups was statistically non-significant: $\mathrm{SM}$ $(\mathrm{p}=0.13), \mathrm{NM}(\mathrm{p}=0.41), \mathrm{IM}(\mathrm{p}=0.61)$, and TM $(\mathrm{P}=0.62)$ respectively.

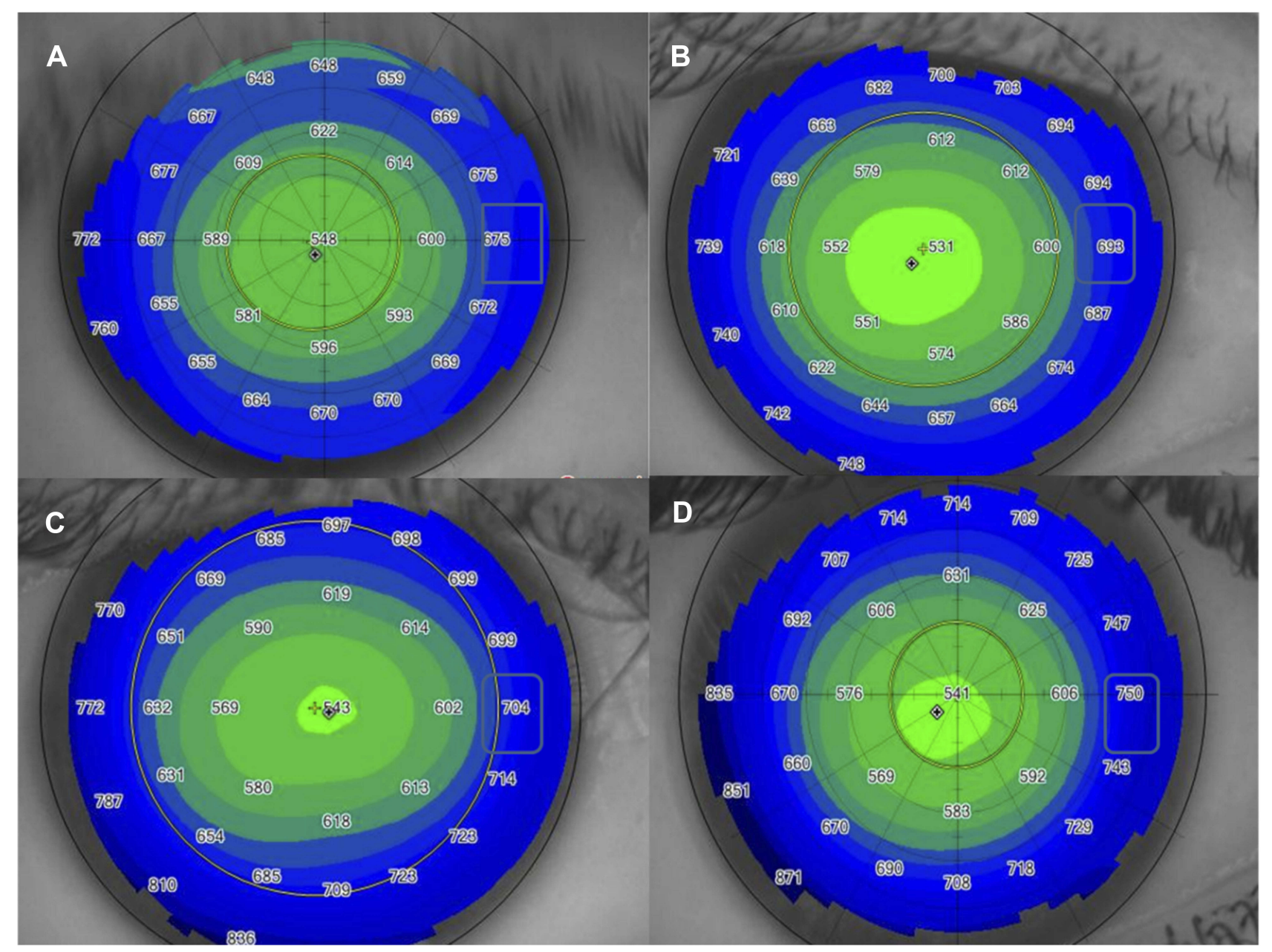

Figure I Corneal pachymetry map showing: (A) Normal pachymetry map (control group), (B) SLE patient with SLEDAI score 5, (C) SLE patient with SLEDAI score I6 and (D) SLE patient with SLEDAl score 32. Progressive peripheral nasal thickening is noted with higher SLEDAI scores. 
Table 3 Correlation Between Pachymetry Readings at Different Corneal Zones and the SLEDAI Score

\begin{tabular}{|l|l|l|}
\hline & \multicolumn{2}{|l|}{ SLEDAI Score } \\
\hline Mean CCT (microns) & Correlation Coefficient & -0.021 \\
& P value & 0.914 \\
\hline Thinnest location (microns) & Correlation Coefficient & -0.061 \\
& P value & 0.759 \\
\hline SM (microns) & Correlation Coefficient & 0.076 \\
& P value & 0.700 \\
\hline NM (microns) & Correlation Coefficient & 0.325 \\
& P value & 0.091 \\
\hline IM (microns) & Correlation Coefficient & 0.149 \\
& P value & 0.448 \\
\hline TM (microns) & Correlation Coefficient & 0.047 \\
& P value & 0.812 \\
\hline SP (microns) & Correlation Coefficient & -0.003 \\
& P value & 0.988 \\
\hline NP (microns) & Correlation Coefficient & 0.407 \\
& P value & 0.031 \\
\hline IP (microns & Correlation Coefficient & 0.166 \\
& P value & 0.400 \\
\hline TP (microns) & Correlation Coefficient & -0.079 \\
& P value & 0.688 \\
\hline
\end{tabular}

Abbreviations: CCT, central corneal thickness; SM, superior mid-periphery; NM, nasal mid-periphery; IM, inferior mid-periphery; TM, temporal mid-periphery; SP, superior periphery; IP, inferior periphery; NP, nasal periphery; TP, temporal periphery.

\section{Corneal Thickness at Periphery \\ In the SLE Group}

The mean corneal thickness at the superior peripheral (SP) point was $666.6 \pm 49$ microns, nasal periphery (NP) was 686.7 \pm 45.3 microns, inferior periphery (IP) was $679.1 \pm 41.3$ microns, and the temporal periphery (TP) was $661 \pm 43.6$ microns.

\section{In the Control Group}

The mean corneal peripheral thickness at SP point was $658.3 \pm 38.9$ microns, NP was $631.6 \pm 36.9$ microns, IP was $619.9 \pm 32.9$ microns, and TP was $633.4 \pm 42.9$ microns.

The difference between the mean peripheral thickness at corresponding superior points was statistically nonsignificant $(p=0.47)$, while the difference between corresponding points in the nasal, inferior and temporal periphery was all statistically highly significant: NP $(p<0.001)$, IP $(\mathrm{p}<0.001)$, and TP $(\mathrm{P}=0.018)$ respectively, with SLE patients having significantly thicker corneal periphery than controls
(Table 2). This was still highly significant after sex adjustment between SLE patients and controls NP $(\mathrm{p}<0.001)$, IP $(\mathrm{p}<0.001)$ and TP $(\mathrm{p}=0.02)$.

\section{Best-Corrected Visual Acuity BCVA}

In the SLE group, the mean BCVA was $0.66 \pm 0.2$ while in the control group it was $0.82 \pm 0.1$. Mean BCVA was lower in the SLE group than in the control group, $\mathrm{P}=0.002$.

\section{Correlation Between Studied Variables and Disease Activity}

A positive correlation of high statistical significance was found between the corneal thickness at the nasal periphery and the disease activity SLEDAI score $(\mathrm{r}=0.4, \mathrm{p}=0.03)$. Another positive correlation $(r=0.3)$ was found between the nasal mid-peripheral corneal thickness and the SLEDAI score. However, this correlation was not statistically significant $(\mathrm{p}=0.09)$. The latter findings highlight the presence of peripheral nasal corneal thickening which may correlate with disease activity in SLE patients. The authors noted progressively higher peripheral nasal thickness readings with higher SLEDAI scores as seen in (Figure 1) and this was a consistent finding (Table 3).

\section{Discussion}

Corneal changes in systemic disease have generally been little studied. It is expected that, being made primarily of type 1 collagen, the corneal stroma, would be affected by connective tissue diseases (CTDs) ${ }^{14,15}$

In our study, we found that SLE patients have statistically significant thinner central corneal pachymetry as well as lower thinnest pachymetry when compared to normal subjects. This significance decreased after adjustment for sex as females tend to have thinner corneal thickness, and lupus is a disease with female predilection. This relative central thinning, however, can be explained by the fact that pathologically CTDs are suggested to be a local antigenantibody reaction that triggers a number of cellular and biochemical responses, resulting in lysis of corneal collagen and subsequent corneal thinning. ${ }^{15}$ However, we cannot exclude other contributing factors as the pathogenesis of SLE is complex, multi-factorial and not yet fully understood. ${ }^{16}$

In the current study, the authors found statistically significant peripheral corneal thickening in the nasal, inferior and temporal quadrants when compared to controls. The difference was still significant even after gender adjustment. 
Nevertheless, there was no significant difference in peripheral corneal thickness in the superior quadrant between the two groups. The authors were intrigued by this finding, while other studies support the fact that peripheral corneal thickening may occur in SLE, they mostly explain this finding by immune complex deposition at the conjunctival, limbal and ciliary body blood vessels as well as at the basement membrane of the cornea. ${ }^{17}$ The authors agree with the theory of autoantibodies formation in SLE and the subsequent immune complex deposition which takes place at the corneal periphery causing subsequent thickening. However, this does not explain why the superior corneal periphery was "selectively spared" from this peripheral thickening. In their own theory, the authors postulate that corneal peripheral thickening could partially be a reaction to UVR as part of the general photosensitivity that happens with SLE. The upper part of the corneal being always "covered by the upper eye lid" could be relatively protected from direct sunlight and thus UVR exposure. In their theory, the authors built on the observation that pterygium - for example occurs almost always nasally and is also postulated to be related to UVR exposure despite having a totally different pathogenesis than that of SLE. ${ }^{18-20}$

Statistically significant flatter posterior corneal surface was also found in our SLE patients in the $8 \mathrm{~mm}$ zone. It is not known, however, if this finding is a cause or a result of the peripheral corneal thickening we found in this study. Whether this peripheral posterior corneal flattening was due to immune complex deposition that pushed the posterior corneal surface backwards, or it is primarily related to the pathogenesis of disease and contributed - as a causative factor - to the peripheral corneal thickening is a point that needs further research.

In this study, the nasal peripheral corneal thickness significantly correlated with systemic disease activity as indicated by the SLEDAI score.

No significant anterior corneal curvature changes were found in our study as opposed to patients with systemic sclerosis who were found to have steeper corneas than normal subjects. ${ }^{21}$

Similar to our study, Gunes A et al found significantly decreased mean CCT and mean corneal volume when compared to healthy controls in patients with ankylosing spondylitis (AS). The same authors reported significantly thinner central pachymetry and increased peripheral thickness in patients with rheumatoid arthritis (RA). ${ }^{22,23}$

For better understanding of the changes in corneal biomechanics, it is important to correlate them with pachymetry. Kazutaka et al in an earlier study showed that corneas with thinner CCT exhibit lower corneal hysteresis. ${ }^{24}$ Moreover, Yazici et al in a recent study found that corneal hysteresis $(\mathrm{CH})$ and corneal resistance factor (CRF) were significantly lower in SLE patients than in a control group. ${ }^{7}$ Building on these findings, our results can be used for future studies to understand the change in biomechanical properties in SLE patients.

In conclusion, the cornea being a structure rich in collagen is especially vulnerable to connective tissue disease. Central corneal thinning seems to be a common finding between SLE, AS, and RA, whereas peripheral corneal thickening may be unique to SLE. The nasal peripheral thickness is positively correlated to systemic disease activity.

If - by future larger studies - this correlation proved to have high specificity, it could accordingly be used to confirm disease activity. Based on the authors proposed theory of corneal photosensitivity in SLE patients, it could be beneficial and advisable for lupus patients to wear sunglasses with high UV protection during times of sunlight exposure. Our reported corneal parameter changes should be taken into consideration when consulting with SLE patients who come for refractive surgery. They should also be taken into consideration when performing ORA in SLE patients who are glaucoma suspects, as corneal thinning may give lower intraocular pressure readings.

\section{Data Sharing Statement}

All data and supplementary data are available with the corresponding author upon request.

\section{Acknowledgment}

The authors would like to thank colleagues in the rheumatology department (Wael Sadek, Ghada S. El-Azkalany and Wafaa Gaber) for their enthusiastic collaboration in the assessment of disease activity index.

\section{Disclosure}

The authors report no financial interest in any of the material used in this study.

\section{References}

1. Tikly M, Navarra SV. Lupus in the developing world - is it any different? Best Practice and Res Clin Rheum. 2008;22(4):643-655.

2. D'Cruz DP, Khamashta MA, Hughes GR. Systemic lupus erythematosus. Lancet. 2007;369(9561):587-596. doi:10.1016/S01406736(07)60279-7

3. Jensen JL, Bergem HO, Gilboe IM, Husby G, Axéll T. Oral and ocular sicca symptoms and findings are prevalent in systemic lupus erythematosus. J Oral Pathol and Med. 1999;28(7):317-322. doi:10.1111/j.1600-0714.1999.tb02047.x 
4. Palejwala NV, Walia HS, Yeh S. Ocular manifestations of systemic lupus erythematosus: a review of the literature. Autoimmune Dis. 2012;2012:290898. doi:10.1155/2012/290898

5. Messmer EM, Foster CS. Vasculitic peripheral ulcerative keratitis. Surv Ophthalmol. 1999;43(5):379-396. doi:10.1016/S0039-6257(98)00051-4

6. Varga JH, Wolf TC. Bilateral transient keratoendotheliitis associated with systemic lupus erythematosus. Ann Ophthalmol. 1993;25(6):222-223.

7. Yazici AT, Kara N, Yuksel K, et al. The biomechanical properties of the cornea in patients with systemic lupus erythematosus. Eye. 2011;25(8):1005-1009. doi:10.1038/eye.2011.99

8. Nemet AY, Vinker S, Bahar I, Kaiserman I. The association of keratoconus with immune disorders. Cornea. 2010;29(11):1261-1264. doi:10.1097/ICO.0b013e3181cb410b

9. Zandman-Goddard G, Solomon M, Rosman Z, Peeva E, Shoenfeld Y. Environment and lupus-related diseases. Lupus. 2012;21:241-250. doi:10.1177/0961203311426568

10. Hochberg MC. Updating the American college of rheumatology revised criteria for the classification of systemic lupus erythematosus. Arthritis Rheum. 1997;40(9):1725. doi:10.1002/(ISSN)1529-0131

11. Bombardier C, Gladman DD, Urowitz MB, Caron D, Chang CH; Derivation of the SLEDAI. A disease activity index for lupus patients. The committee on prognosis studies in SLE. Arthritis Rheum. 1992;35:630-640. doi:10.1002/art.1780350606

12. Hirnschall N, Crnej A, Gangwani V, Findl O. Effect of fluorescein dye staining of the tear film on scheimpflug measurements of central corneal thickness. Cornea. 2012;31(1):18-20. doi:10.1097/ICO.0b013e31821eea97

13. Feng Y, Varikooty J, Simpson TL. Diurnal variation of corneal and corneal epithelial thickness measured using optical coherence tomography. Cornea. 2001;20(5):480-483. doi:10.1097/00003226200107000-00008

14. Fontes BM, Ambrósio R Jr, Jardim D, Velarde GC, Nosé W. Corneal biomechanical metrics and anterior segment parameters in mild keratoconus. Ophthalmology. 2010;117:673-679. doi:10.1016/j. ophtha.2009.09.023
15. Hyams SW, Kar H, Neumann E; Blue sclerae and keratoglobus. Ocular signs of a systemic connective tissue disorder. $\mathrm{Br}$ J Ophthalmol. 1969;53:53-58. doi:10.1136/bjo.53.1.53

16. Davies JB, Rao PK. Ocular manifestations of systemic lupus erythematosus. Curr Opin Ophthalmol. 2008;19:512-518. doi:10.1097/ ICU.0b013e3283126d34

17. Reeves JA. Keratopathy associated with systemic lupus erythematosus. Arch Ophthalmol. 1965;74:159-160. doi:10.1001/archopht.1965.0097 0040161005

18. Golu A, Gheorghişor I, Bălăoiu AT, et al. The effect of ultraviolet radiation on the cornea - experimental study. Rom J Morphol Embryol. 2013;54(4):1115-1120.

19. Moran DJ, Hollows FC. Pterygium and ultraviolet radiation: a positive correlation. $\mathrm{Br} \quad J$ Ophthalmol. 1984;68(5):343-346. doi:10.1136/ bjo.68.5.343

20. Threlfall TJ, English DR. Sun exposure and pterygium of the eye: a dose-response curve. Am J Ophthalmol. 1999;128(3):280-287. doi:10.1016/S0002-9394(99)00161-0

21. Nagy A, Rentka A, Nemeth G, et al. Corneal manifestations of systemic sclerosis. Ocul Immunol Inflamm. 2018:1-10. doi:10.1080/ 09273948.2018 .1489556

22. Gunes A, Inal EE, Tok L, Tok O. Assessment of corneal parameters with scheimpflug imaging in patients with ankylosing spondylitis. Semin Ophthalmol. 2017;32(3):276-280. doi:10.3109/08820538.2015.1068340

23. Gunes A, Inal EE, Tok L, Tok O. Evaluation of central and peripheral corneal thicknesses in patients with rheumatoid arthritis. Arq Bras Oftalmol. 2015;78(4):236-240. doi:10.5935/0004-2749.20150061

24. Kamiya K, Hagishima M, Fujimura F, Shimizu K. Factors affecting corneal hysteresis in normal eyes. Arch Clin Exp Ophthalmol. 2008;246:1491. doi:10.1007/s00417-008-0864-x
Clinical Ophthalmology

\section{Publish your work in this journal}

Clinical Ophthalmology is an international, peer-reviewed journal covering all subspecialties within ophthalmology. Key topics include: Optometry; Visual science; Pharmacology and drug therapy in eye diseases; Basic Sciences; Primary and Secondary eye care; Patient Safety and Quality of Care Improvements. This journal is indexed on PubMed
Dovepress

Central and CAS, and is the official journal of The Society of Clinical Ophthalmology (SCO). The manuscript management system is completely online and includes a very quick and fair peer-review system, which is all easy to use. Visit http://www.dovepress.com/ testimonials.php to read real quotes from published authors. 